\title{
Malignant mixed mullerian tumour of the endometrium with a synchronous ovarian cancer- a rare association
}

\author{
Sathyavani Chennaihgari*, Padmaja Pidaparthy, Rajnish Samal, Nalini Arun Kumar, \\ Agitha Kumari
}

Department of Obstetrics \& Gynecology, Bangalore Baptist Hospital, Hebbal, Bellary road, Bangalore-560024, Karnataka, India

Received: 17 November 2012

Accepted: 11 December 2012

*Correspondence:

Dr. Sathyavani C.,

E-mail: drsathyac@yahoo.co.in

\begin{abstract}
Synchronous malignancies of genital tract are extremely uncommon especially those with independent histopathologies. We are reporting a rare case with a unique presentation. A 79 year old nulliparous lady presented with foul smelling discharge and bleeding per vaginum. Examination revealed an abdominopelvic mass and a friable polypoidal growth projecting from the cervical os. Biopsy of the polyp indicated mixed malignant mullerian tumour. CT scan revealed a large ovarian tumour with solid and cystic areas indicating ovarian malignancy. During laparotomy patient was also found to have chronic inversion of uterus secondary to the polypoidal growth protruding from the cervix. Histopathology confirmed mixed malignant mullerian tumour of the endometrium and serous cyst adenocarcinoma of the right ovary.
\end{abstract}

Keywords: Synchronous neoplasm, Endometrial MMMT, Epithelial ovarian tumour

\section{INTRODUCTION}

Synchronous malignancy in female genital tract is very rare accounting to about $0.63-1.7 \%$ of all genital malignancies. ${ }^{1}$ Most of the reported cases are tumours with common aetiology e.g. endometrial carcinoma and breast carcinoma (oestrogen dependant), ovarian and breast carcinoma (common gene), endometrial and ovarian endometroid tumours (hormonal field effect). ${ }^{2}$ It is extremely rare to have two malignancies arising from two different cell lines. Here we report a case with two independent malignancies which is very rare.

\section{CASE REPORT}

A 79 year old nulliparous lady presented with history of bleeding per vagina for one week. Systemic examination revealed a hard, non-tender lobulated abdomino-pelvic mass extending up to the umbilicus. There was no hepato-splenomegaly or ascites. Speculum examination revealed a large friable polypoidal mass protruding from cervical os and occupying the whole of the upper vagina. Rectal mucosa and parametrium were free.
Biopsy of the polypoidal mass was reported as malignant mixed mullerian tumour (MMMT). CT scan showed a bulky cervix with a large hypo dense cystic lesion causing thinning of cervix, a large right ovarian cystic mass with two small enhancing solid components within and no evidence of ascites or lymph node enlargement. CT thorax revealed parenchymatous opacities in the lung suggestive of metastasis. CA 125 was $38.7 \mathrm{U} / \mathrm{ml}$.

A provisional diagnosis of stage IV uterine sarcoma (MMMT) with a coexisting ovarian malignancy was made. Palliative treatment was planned after consulting the oncologist. But as the patient opted for symptom relief only, she was taken up for palliative surgery.

Intra operatively minimal haemorrhagic ascites was present. Chronic inversion of the uterus due to the large sub-mucous fibroid polyp was noted. Right ovarian cyst of $8 \times 8 \mathrm{cms}$ with an intact capsule and variable consistency was noted (Figure $1 \& 2$ ). Total abdominal hysterectomy with right salpingo ovariotomy and left salpingo oophorectomy was done. Postoperative period was uneventful and patient recovered well. 


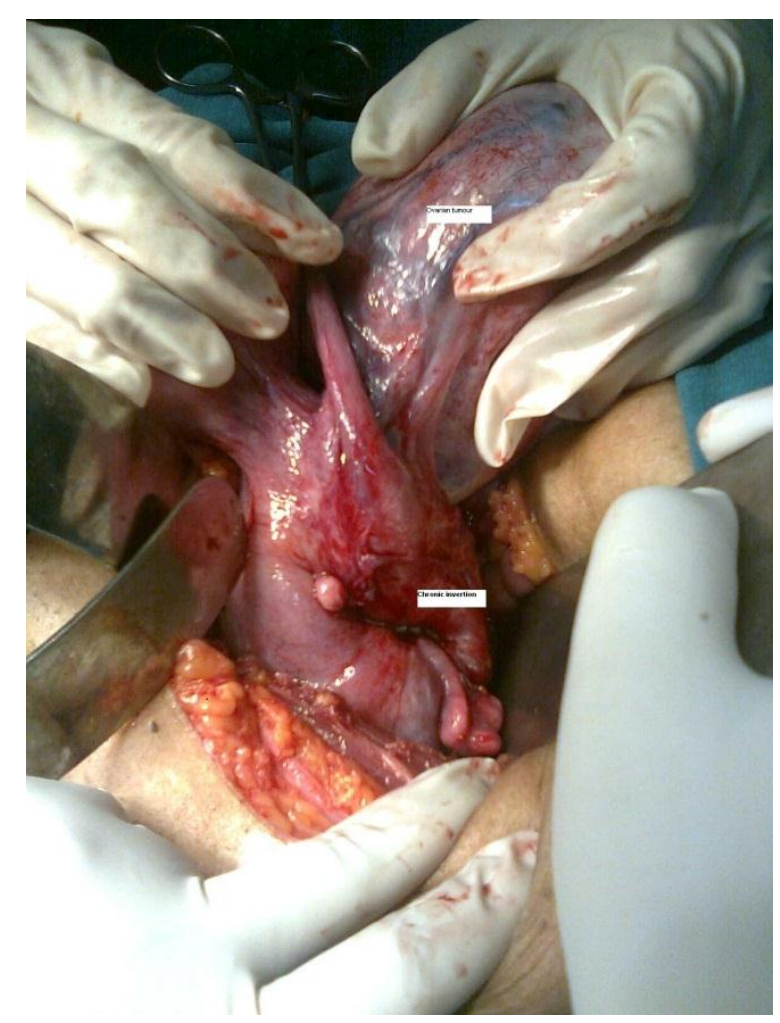

Figure 1: Chronic inversion of uterus and ovarian tumour.

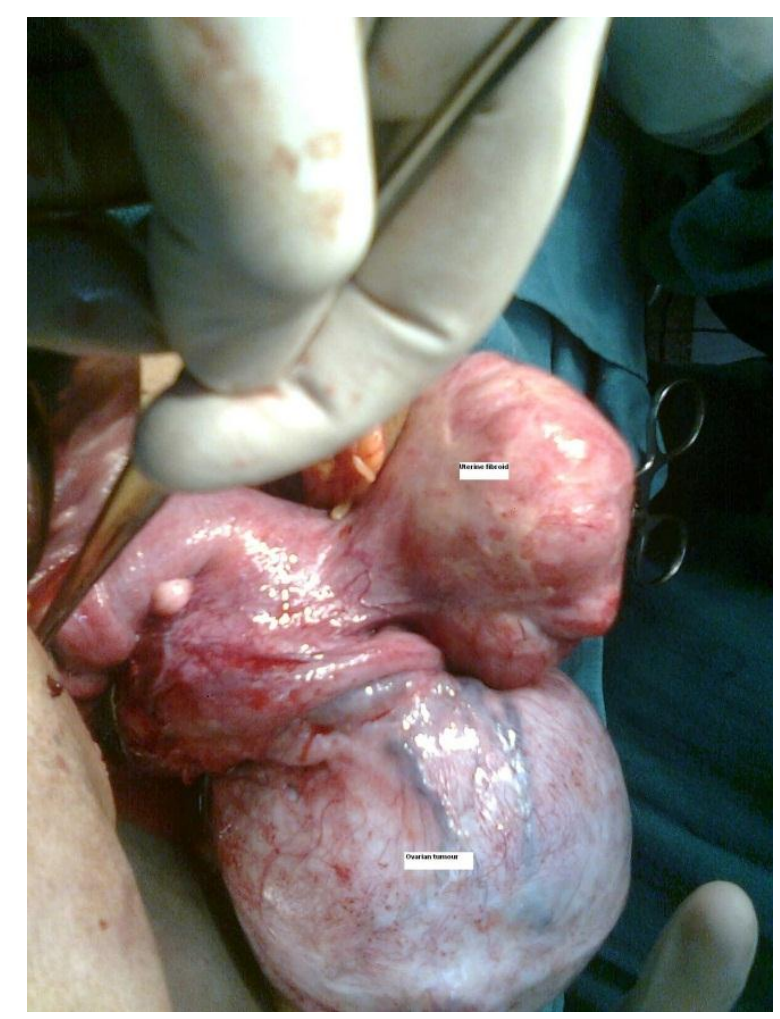

Figure 2: Uterine fibroid and ovarian tumour.

Histopathology of the surgical specimen confirmed high grade undifferentiated uterine sarcoma most likely malignant mullerian tumour and co existing serous cystadenocarcinoma of right ovary. Peritoneal cytology was positive for malignant cells.

Patient had symptom free comfortable life for more than one year and then lost to follow up.

\section{DISCUSSION}

The incidence of synchronous genital malignancy is reported to vary between $0.63 \%-1.7 \%$ of all genital malignancies. The most frequently observed association is between neoplasm of ovary and endometrium. $(51.7 \%)^{2}$

It is important to distinguish multiple primary neoplasms from metastatic disease. The criteria for identification of synchronous primary cancers include either the detection of different histological types, or minor criteria like both tumours confined to primary sites with no direct extension between tumours. Our case with two unrelated tumours fulfils these criteria.

MMMT arising from the female genital tract is a rare disease. Simultaneous presence of another coexisting carcinoma is extremely rare and review of literature has revealed only one case with similar co-existence. ${ }^{1-3}$ However synchronous and metachronous tumours are more commonly seen with extragenital MMMTs. ${ }^{4}$

In a review of the English literature since 1955, only 49 cases of extragenital MMMT have been reported. Sixteen out of these $49(32.7 \%)$ extragenital MMMTs, were associated with synchronous or metachronous colonic cancer or gynaecologic malignancy and serous carcinoma of the peritoneum. Hence a detailed examination of the genital tract must be done before and during the surgery. ${ }^{4}$

Surgical treatment is the treatment of choice, as it contributes significantly to the diagnosis of synchronous lesions. Chemotherapeutic agents are chosen to target the most aggressive tumour of the two. In our patient drugs targeting MMMT like adriamycin, dacarbazine, paclitaxel/carboplatin with ifosfamide would have been useful.

Prognosis in patients with synchronous malignancies is reported to be more favourable than metastatic individual tumours. However malignant mixed mullerian tumour is associated with unfavourable prognosis.

This case is reported due to the rarity of occurrence. Though, treatment is customised according to patient's wishes, she had a symptom free life for one year. In a younger patient, radical surgery and chemotherapy would have helped.

\section{CONCLUSION}

Synchronous gynaecologic tumours may co-exist. Improved survival would be obtained if accurate 
diagnosis and aggressive treatment, including cytoreductive surgery and chemotherapy are offered.

\section{REFERENCES}

1. Tong SY, Lee YS, et al. Clinical analysis of synchronous primary neoplasms of the female reproductive tract. Eur J Obstet Gynecol Reprod Biol 2008;136:78-82.

2. Ayhan A, Yalçin OT, Tuncer ZS, Gürgan T, Küçükali T. Synchronous primary malignancies of the female genital tract. Eur J Obstet Gynecol Reprod Biol 1992;45:63-6.

3. Soliman PT, Slomovitz BM, Broaddus RR, Sun CC, Oh JC, Eifel PJ, et al. Synchronous primary cancers of the endometrium and ovary: a single institution review of 84 cases. Gynecol Oncol 2004;94:456-62.

4. Huang CC, Ma CJ, Huang WT, Chan TF, Wang JY. Primary malignant mixed Müllerian tumor arising from the mesorectum with a synchronous ovarian cancer: a case report and review of the literature. J Med Case Rep 2011;5:15.

DOI: $10.5455 / 2320-1770$. ijrcog000512

Cite this article as: Sathyavani C, Pidaparthy P, Samal R, Kumar NA, Kumari A. Malignant mixed mullerian tumour of the endometrium with a synchronous ovarian cancer- a rare association. Int $\mathbf{J}$ Reprod Contracept Obstet Gynecol 2012;1:52-4. 